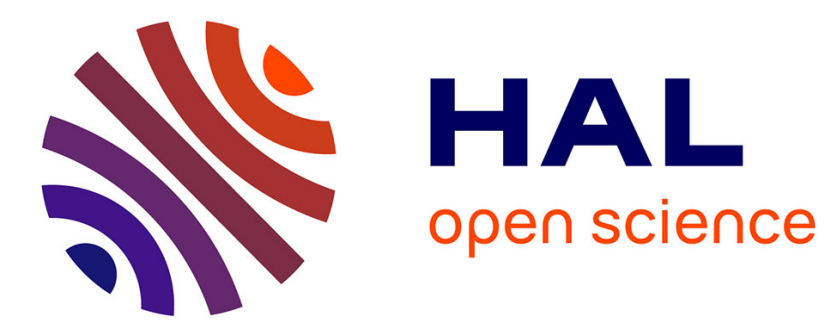

\title{
La justice royale et les juifs dans l'espace aragonais, quels enjeux ?
}

Claire Max Soussen

\section{To cite this version:}

Claire Max Soussen. La justice royale et les juifs dans l'espace aragonais, quels enjeux ?. Le Moyen Age. Revue d'histoire et de philologie, 2012, CXVIII (2), 10.3917/rma.182.0411 . hal-01704251

\section{HAL Id: hal-01704251 \\ https://hal.science/hal-01704251}

Submitted on 9 Feb 2018

HAL is a multi-disciplinary open access archive for the deposit and dissemination of scientific research documents, whether they are published or not. The documents may come from teaching and research institutions in France or abroad, or from public or private research centers.
L'archive ouverte pluridisciplinaire HAL, est destinée au dépôt et à la diffusion de documents scientifiques de niveau recherche, publiés ou non, émanant des établissements d'enseignement et de recherche français ou étrangers, des laboratoires publics ou privés. 


\title{
« La justice royale et les juifs dans l'espace aragonais, quels enjeux ? », Claire Soussen.
}

\author{
Le Moyen Âge 2/2012, tome CXVIII, p. 411-432
}

\section{Introduction.}

Le pouvoir royal aragonais reconnaît à la minorité juive dans ses territoires une autonomie de gestion interne : à l'intérieur des communautés locales, l'entité juive jouit de privilèges qui lui permettent de prendre des décisions et de les faire appliquer tout en respectant les lois de l'État qui l'accueille. De la sorte, le principe talmudique Dina de Malkhuta Dina formulé au III $^{\mathrm{e}}$ siècle y est appliqué dans son sens le plus plein et les juifs peuvent s'épanouir dans la diaspora aragonaise. Sans aller jusqu'à parler d'un État dans l'État ${ }^{1}$, l'entité juive dans la Couronne d'Aragon bénéficie d'infrastructures et de champs de compétences étendus ${ }^{2}$. Les communautés locales disposent d'un personnel divers et spécialisé pour assumer les tâches nécessaires à leur fonctionnement et, par ordonnances, le roi assure l'homogénéisation et la normalisation des aljamas, toutes organisées sur le même modèle. Un élément en particulier semble emblématique de la façon dont s'organisent les relations entre le roi et les juifs présents dans ses territoires : la justice. La justice en effet met en jeu l'autorité du roi, l'autonomie juive, et plus largement les tentatives d'affirmation des pouvoirs concurrents du roi $^{3}$. L'exercice de la justice sur les juifs est une des facettes de la relation particulière qui unit le roi à l'entité juive dans ses États. De fait, l'exception juive qui fait que le roi considère les juifs comme $"$ siens $^{4}$ » se traduit par une organisation judiciaire

\footnotetext{
${ }^{1}$ Voir L. FinKelstein, Jewish self-government in the Middle Ages, New York, 1924, p. 8. Voir aussi pour un aperçu plus nuancé mais dans la même ligne, D. BIALE, Power and Powerlessness in Jewish History, New York, 1986, p. 56 ; M. Lorberbaum, Politics and the Limits of Law. Secularizing the Political in Medieval Jewish Thought, Stanford, 2001, p. 98.

${ }^{2}$ Voir Y. T. Assis, The Golden Age of Aragonese Jewry: Community and Society in the Crown of Aragon, 1213-1327, London-Portland, 1997, p. 9. Les privilèges accordés à l'entité juive dans ses États par le roi d'Aragon seraient la contrepartie d'un «échange de bons procédés » selon lequel [...] los jodios siervos son del sennor Rey et sienpre a la real bolsa son co(n)tados, comme l'expriment les Fueros de Teruel au début du XIII ${ }^{\mathrm{e}}$ siècle. Il n'est pas lieu ici de discuter le thème de la servitude juive -analysé de façon passionnante par D. ABULAFIA- sur lequel débouche cet extrait cité, Nam iudei servi regis sunt, et semper fisco regio deputati : The Jews in the municipal Fuero of Teruel (1176-7), Jews, Muslims and Christians in and around the Crown of Aragon, Essays in Honour of Prof. Elena Lourie, éd. H. J. HAMES, Leiden-Boston, 2004, p. 99, mais de comprendre en quoi cette formule illustre la relation particulière qui unit le roi d'Aragon aux juifs dans ses États. ${ }^{3}$ Sur la concurrence que se livrent le roi et les seigneurs autour de la justice voir P. A. PORRAS ARBOLEDAS, E. RAmirez VAQUero et F. SABATÉ i Curull, Historia de España VIII, La época medieval : administración y gobierno, Madrid, 2003, p. 389-390.

${ }^{4}$ Voir J. AMADOR DE LOS Rios, Historia de los Judios de España y Portugal, Madrid, 1875-1876, rééd. 1984, Tome 1, p. 407, rappelle les principes exposés dans les Fueros de Aragon selon lesquels les juifs sont sous la seule autorité du roi et ne peuvent avoir que le roi pour juge. De même, les Furs de Valencia, cités par l'auteur p. 416, exposent les mêmes principes. Ces principes étant établis, le principal privilège accordé aux juifs en matière de justice, est le fait qu'ils soient autorisés à juger eux-mêmes les affaires entre juifs. Voir aussi ABULAFIA, Nam iudei servi regis sunt, p. 117-118 en ce qui concerne la règle en vigueur dans les affaires mettant en cause des juifs et des chrétiens et pas seulement des juifs.
} 
spécifique, reposant sur un équilibre subtile entre respect de la $H_{a l a k h a^{5}}$-la loi juive- et soumission à la justice royale ${ }^{6}$. Le pouvoir royal entérine et rappelle à ses sujets juifs, lorsqu'ils l'oublient, leur devoir d'observance à l'égard de la loi juive qui prime dans la plupart des affaires lorsque s'impose le recours au droit. C'est en fait seulement lorsque justice est rendue que s'impose l'autorité du roi, qui se traduit par la perception des droits de justice, enjeu économique fondamental de la question judiciaire. Cette mécanique bien huilée fonctionne le plus souvent sans accroc, la répartition des compétences répondant à une réciprocité d'intérêts bien comprise, sauf lorsque s'interposent les prétentions de tiers.

\section{La justice et les juifs : quel fonctionnement ?}

Dans les relations avec les chrétiens comme dans leur attitude à l'égard du pouvoir, les juifs sont soumis au droit commun, en revanche, dans les affaires internes à la communauté juive, ils ont le privilège d'être régentés par la Halakha.

\section{1) La Halakha: Une référence légale dans l'espace aragonais}

La Halakha est la loi juive, l'ensemble législatif rassemblé à partir des règles formulées dans la Torah et des développements auxquelles elles ont donné lieu dans le Talmud avec la Michna et la Guemara'. Tout juif a l'obligation de se conformer à cette loi que l'on appelle aussi loi de Moïse. Il s'agit d'une loi religieuse, mais elle a également une dimension pratique et politique. C'est le caractère obligatoire et imprescriptible de cette loi qui obligea les autorités rabbiniques à adopter une position théorique à l'égard de la loi en vigueur au sein des territoires dans lesquels elles se trouvaient. Il fallait pouvoir continuer à observer la Halakha tout en n'étant pas hors-la-loi du pays d'accueil. Ce double impératif conduisit à la formulation par un maître du Talmud, maître Samuel, de la règle Dina de Malkhute Dina, entérinant le respect par les juifs de la loi en vigueur là où ils se trouvent, tant qu'elle n'entre pas en contradiction avec la loi juive ${ }^{8}$. Pour l'époque qui nous occupe, le principe en est confirmé par Salomon Ben Adret ${ }^{9}$, autorité juive de Barcelone. Le modus vivendi ainsi dégagé, est entériné par le pouvoir temporel dans la Couronne d'Aragon.

\footnotetext{
${ }^{5}$ Le respect de la Halakha est en effet la condition sine qua non de l'existence des juifs en diaspora ; condition sinon partout explicite, du moins implicite à l'intérieur des communautés en Occident

${ }^{6}$ Voir LORBERBAuM, Politics and the limits of the Law, p. 98.

${ }^{7}$ Voir E. Gugenheim, Les portes de la Loi. Études et Responsa, Paris, 1982, p. $50 \mathrm{~s}$.

${ }^{8}$ Ibid., p. 267.

${ }^{9}$ Voir RASHBA Responsum $n^{\circ} 637,1^{\text {ère }}$ partie, éd. CD-Rom Responsa Project de 1'Université Bar Ilan.
} 
En 1270 et 1271 Jacques Ier adresse à deux juifs des lettres confirmant leur fonction de juge ${ }^{10}$ et donnant des précisions sur les textes qui doivent leur servir de référence. La première concerne l'aljama de Lérida, la seconde l'aljama de Saragosse. Dans le premier cas, le roi confie au juif Yoci Azday la fonction de «Rabbin sur les juifs de Lérida avec mandat dans l'Ancien Testament, et juge de l'aljama des juifs de Lérida selon la loi des juifs,...tu auras avec toi deux juifs parmi les meilleurs et les plus sages... ${ }^{11}$. Les mentions de azuna iudeorum et lex vetus font donc explicitement référence à la loi juive et à l'Ancien Testament désigné sous l'expression d'“Ancienne Loi". Ces hommes sont désignés pour leur savoir, conformément à la coutume en vigueur depuis les temps bibliques, où les sages avaient aussi un rôle dirigeant. Le rabbin de la communauté, expert dans la Halakha, est ainsi chargé d'assumer l'exercice de la justice en se faisant assister par deux hommes dont l'honnêteté est la meilleure garantie.

Le second document est produit dans un contexte beaucoup plus conflictuel puisque l'homme désigné par le roi pour exercer la fonction de juge est contesté par des membres de l'aljama de Saragosse. Sans entrer dans les détails de l'affaire, on voit bien ici encore que la loi juive est l'instrument de régulation à l'intérieur de la communauté : «...nous avons concédé à Salomon notre alfaquim...qu'il puisse juger, décider et poursuivre toutes affaires des juifs de Saragosse et du royaume d'Aragon par ses officiers, en fonction de la loi des juifs $\gg{ }^{12}$. C'est bien en fonction de la Halakha que le représentant du roi à Saragosse exerce sa charge de juge. Il faut souligner par ailleurs, que bien que contemporaines, ces lettres reflètent l'évolution évoquée plus haut en ce qui concerne les responsabilités au sommet de l'aljama, c'est-à-dire le passage d'une responsabilité assumée par l'autorité savante incarnée par le rabbin, à une responsabilité assumée par l'autorité politique ou économique incarnée par l'alfaquim du roi -qui est souvent un membre de l'oligarchie juive locale-. Cette évolution se fait le plus souvent sans heurt, mais ce n'est pas le cas ici où la légitimité de l'alfaquim est remise en cause, sa nomination s'étant faite hors de la voie démocratique et collégiale habituellement suivie dans le cadre des aljamas pour la désignation des juges.

\footnotetext{
${ }^{10}$ BArcelone, Archives de la Couronne d'Aragon (ACA), Chancellerie Royale, reg. 16, fol. 202, Valence 28.07.1270, ff. 261v-262, Valence 24.04.1271. Ces documents sont recensés par J. RÉGNÉ, History of the Jews in Aragon, Regesta and Documents 1213-1327, rééd. Y. T. Assis, Jerusalem, 1978, $\mathrm{n}^{\circ \mathrm{s}} 446$ et 461.

${ }^{11}$ BARCELONE, ACA, reg. 16 fol. 202r, Valence 28.07.1270 : [...] Rab super iudeis Ilerde in mandatis legis veteris et [...] iudex aliame iudeorum Ilerde...secundum azunam iudeorum et habeas de cetero tecum duos iudeos de melioribus et sapientioribus [...]

${ }^{12}$ Ibid. ff. 261v-262, Valence 24.04.1271 : [...] nos concessissemus Salomoni alfaquimo nostre [...] quod ipse omnes causas iudeorum Cesarauguste et regni Aragone possit iudicare decidere et exsequi per suos officiales secundum legem iudeorum.
} 


\section{2) L'administration de la justice.}

Dans une ordonnance adressée par Pierre III aux aljamas de Catalogne en 1280, le roi définit les modalités de leur organisation et de leur fonctionnement interne, et précise surtout leur organisation en matière de justice. Les agents de l'aljama doivent: «...connaître les crimes, questions, controverses, requêtes qui surviennent entre juifs et juives et entre chrétiens et juifs ...ils peuvent condamner et punir les juifs et les juives de cette aljama et des localités qui dépendent de la collecte de cette aljama...des blessures, injures, sottises, maléfices et tout ce qu'ils pourraient être amenés à éclaircir $»^{13}$. L'essentiel de la responsabilité des agents de l'aljama consiste donc à assumer la justice à l'intérieur de la communauté. Ces hommes sont chargés d'arbitrer les conflits et différends survenus entre juifs ou entre juifs et chrétiens. Ce sont à la fois les instances devant lesquelles porter plainte et les arbitres, dépositaires et exécuteurs de la justice. Le roi précise également quelle doit être la référence de jugement pour ces hommes : «Et ceux-ci doivent être punis par les hommes probes selon le droit hébraïque ${ }^{14}$. Par ailleurs, leurs attributions leur donnent sur les coupables le pouvoir de : «... les prendre et les faire prendre et procéder à une peine d'exil contre eux et ils peuvent aussi faire des statuts et des interdictions sur leurs faits et gestes et décréter la justice, des alatmas et nitduis $»^{15}$. Les juges ont donc à leur disposition un éventail de mesures pour faire respecter la justice ou redresser les déviants : certains traditionnels tels l'emprisonnement ou l'exil, d'autres plus spécifiques tels l'anathème ou l'excommunication, désignés par les termes hébraïques alatma et nitdui. Le roi précise ensuite les modalités du passage de la justice juive à la justice du royaume : «...que les juifs qui sont faits prisonniers soient transmis à nos bayles et que toutes les peines auxquelles les hommes probes et autres juifs les ont condamnés en matière criminelle ou en matière civile soient précisées à nos bayles qui les feront appliquer pour nous $»^{16}$. Chaque aljama fonctionne comme une instance autonome, néanmoins, elles peuvent communiquer et adopter des décisions communes ce qui permet

\footnotetext{
${ }^{13}$ Ibid. reg. 44 ff. 187v-188, Tarragone 31.01.1280 ; RÉGNÉ, History of the Jews in Aragon, p. 149, n 823 : [...] cognoscere crimines, questiones, controversias querimonias qui vertantur inter iudeis iudeas, vel inter christanum et iudeum, de peticionibus christianorum et possint corrigere condempnare et punire iudeos et iudeas dicte aljame, vel locorum qui sunt de collecta ipsius aliame [...] de percussionibus, verbis iniuriosis, stulticiis, maleficiis et omnibus aliis in quibus deliquerint.

${ }^{14}$ Ibid. : [...] vel eisdem probis hominibus visum fuerit ipsos debere puniri vel corrigi secundum ius hebraicum.

${ }^{15}$ Ibid. : [...] eos capere et capi facere et procedere ad penam exilii contra eos, possint etiam facere statuta et prohibitiones districtus et ordinaciones super gestibus et actibus eorum et ponere vetita et alatmas et nitdui.

${ }^{16}$ Ibid. : Vero concessionem facimus in hunc modum quod iudeos quos ceperint teneantur tradere baiulis nostris et omnes penas in quibus predicti probi homines aliquem vel aliquos iudeos condempnaverint sive criminaliter sive civiliter teneantur denunciare baiulis nostris et ipsi baiuli nostri exequentur incontinenti et habeant ipsas penas pro nobis.
} 
d'assurer l'intégrité et l'unité de la personnalité juive dans les territoires aragonais ${ }^{17}$. Le fonctionnement des institutions est simplifié et normalisé, le roi adopte une politique homogène à l'égard de l'entité juive. Sur le terrain, le bayle ou représentant du roi, doit faire appliquer les décisions de justice adoptées par les hommes probes de la communauté, qui, par ailleurs, ne peuvent unilatéralement contester et modifier les décisions prises ${ }^{18}$. Il y a de ce fait collaboration et répartition des tâches entre agents juifs et chrétiens chargés de la justice.

Cette ordonnance est fondamentale à plusieurs titres : elle décrit le fonctionnement de la justice de façon précise et se veut de portée générale, donnant cette organisation comme valable sur l'ensemble des terres catalanes. La procédure judiciaire elle-même apparaît donc strictement codifiée. Toutefois certains éléments peuvent varier : le nombre des agents chargés de la justice ${ }^{19}$-le plus souvent trois sur les sept secrétaires de l'aljama- diffère en fonction de l'importance numérique de chaque aljama. Plus une aljama est importante, plus elle requiert de personnel. Le chiffre minimal de deux agents s'explique par la volonté de conserver une organisation collégiale, le chiffre maximal de sept répond à la coutume. Pour certaines décisions importantes, notamment lorsque la peine capitale est en jeu, un collège de dix hommes ou Beth Din, est désigné conformément à la loi juive ${ }^{20}$. Ainsi, en fonction de la gravité des cas jugés, soit on s'en remet aux trois secrétaires de l'aljama -appelés adenantatihabituellement chargés de cet office ${ }^{21}$, soit on convoque un tribunal spécial, le Beth Din qui peut regrouper 3 ou 10 membres. On a vu également que dans certains cas, un seul homme, l'alfaquim, est chargé de rendre la justice. Les principes généraux de la justice royale envers les juifs sont donc homogènes, mais les détails de l'organisation interne aux aljamas varient dans une certaine mesure.

Cette rapide description du fonctionnement de la justice laisse percevoir les enjeux qu'elle recèle.

\footnotetext{
${ }^{17}$ Ibid. L'ordonnance précise en effet: Possint etiam predicte aliame cum aliis aliamis dominacionis nostre concilium et deliberationem iustam habere super predictis.

${ }^{18}$ Ibid. : Similiter ipsi probi homines non possunt diminuere relaxare vel diffinire aliquas penas criminales vel civiles quas ipsi iudei propter culpas vel delicta ipsorum [...] recipere mereantur.

19 Ibid., reg. 206, fol. 32, Barcelone 26.04.1309 ; RÉGNÉ, History of the Jews in Aragon, ${ }^{\circ s} 2898,2899$. Ce document nomme deux hommes, Vitali Maler et Ezcapatus Acarch, secretaris aliame iudeorum Barchinone. Si nous ne pouvons déduire de cette mention qu'il n'y avait que deux secrétaires pour l'aljama de Barcelone, nous voyons cependant qu'il y en avait au moins deux, conformément à ce que requéraient les instructions royales.

${ }^{20}$ BARCELONE, ACA, Chancellerie royale, reg. 21 fol. 126v, Lérida 19.04.1273, RÉGNÉ; History of the Jews in Aragon, p. 94, $\mathrm{n}^{\circ}$ 552. Ici il est prévu la réunion d'un collège de dix hommes socios iudeos usque ad decem, pour juger un blasphémateur qui encourt la peine de mort.

${ }^{21}$ En 1289 une lettre adressée par le roi au bayle de Barcelone rappelle les règles: "Nous voulons que les adenantati de l'aljama des juifs de Barcelone munis des documents hébraïques assignent pour les affaires dessus dites - un contentieux entre plusieurs membres de la communauté - un juif idoine et non suspect des parties, qui entende les motifs des uns et des autres et qui décide et détermine les causes selon le droit hébraïque et la loi des juifs.” BARCELONE, ACA, Chancellerie royale, reg. 80 fol. 95v, Monzon 08.11.1289, RÉGNÉ, History of the Jews in Aragon, p. 360, n 2030 .
} 


\section{Les enjeux de la justice royale à l'égard des juifs.}

Si la Halakha apparaît bien comme le droit de référence pour juger les affaires juives deux systèmes juridiques et judiciaires coexistent dans l'espace aragonais, l'un destiné aux sujets chrétiens du roi d'Aragon, l'autre destiné aux juifs. Or cette coexistence suscite des manoeuvres de la part de justiciables soucieux de voir triompher leur intérêt. En théorie, pour éviter les dysfonctionnements, les champs de compétence des uns et des autres et les règles présidant à l'organisation judiciaire sont fixés de façon homogène à travers les Fueros de Aragon $^{22}$ à partir du milieu du XIII" siècle. Mais, ces règles ne constituent "que" la théorie, et surtout, elles n'évoquent pas la question de l'appel judiciaire ${ }^{23}$.

\section{1) La question de l'appel judiciaire et la garantie de l'autonomie juive.}

En 1272, Jacques Ier adresse une lettre à deux aljamas dans laquelle il précise : «...nous avons concédé aux aljamas des juifs de Gérone et de Bésalu que sur les requêtes, questions et demandes qui surviennent entre eux et d'autres... la partie citée ne puisse faire appel de la décision du juge des juifs en fonction du droit des juifs $»^{24}$. Autrement dit le jugement rendu par les juges juifs a force de loi. Par ailleurs, le roi précise les sanctions auxquelles s'expose tout contrevenant ${ }^{25}$. La question est de savoir qui peut être tenté de faire appel : des juifs au détriment desquels la loi a été appliquée ou bien des chrétiens qui remettent en cause la légitimité de l'application de la loi juive dans des causes dont ils sont partie prenante ${ }^{26}$ ? Cette question apparaît également à travers une lettre adressée par Pierre

\footnotetext{
${ }^{22}$ Voir les articles 107, 114, 134, 275, 320 des Fueros de Aragón : Los fueros de Aragón, según el manuscrito 458 de la Biblioteca nacional de Madrid, éd. G. TILANDER, Lund, 1937. L'article 107 porte sur la validité du témoignage juif en justice, l'article 114 sur l'imprescriptibilité d'un jugement rendu, l'article 134 expose de façon précise une échelle du serment indexée à l'enjeu de la relation. Les articles 138 et 139 décrivent précisément les modalités de l'un et l'autre serments. Les articles 275 et 320 exposent les cas qui peuvent être soumis à la justice, notamment les contentieux de propriété susceptibles d'opposer des juifs et des non-juifs.

${ }^{23}$ Voir W. PAKTER, Medieval Canon law and the Jews, Ebelsbach, 1988, p. 151 : "Comme dans l'antiquité, les cours juives ne fonctionnaient pas sans intervention de l'État. Il était courant pour les autorités séculières et ecclesiastiques [...] de renforcer un Herem [...] Les décisions de justice locale ne prédominaient pas toujours. Un justiciable mécontent pouvait tenter sa chance devant une autorité supérieure, un évêque local ou un roi".

${ }^{24}$ BARCELONE, ACA, Chancellerie royale, reg. 21, fol. 37r, Gérone 15.05.1272, RÉGNÉ History of the Jews in Aragon, $\mathrm{n}^{\circ} 520:[\ldots]$ concesserimus toti aliame iudeorum gerunde et Bisuldine quod super peticionibus, questionibus et demandis [...] quae moveantur inter illos vel aliquos eorumdem pars conventa non possit compelli vel distrigi faciendi ius nisi in posse iudicum iudeorum...et secundum ius iudeorum.

${ }^{25}$ Ibid. : [...] damus licenciam et plenariam potestatem iudeis Gerunde et Bisuldine quod ponant super hiis cessandis [...] excommunicaciones et alatmes quae hebrayce vocantur herem et nitduy [...]. Celui-ci risque donc l'excommunication.

${ }^{26}$ D'après I. EPSTEIN, The responsa of Rabbi Salomon Ben Adreth of Barcelona as a source for the History of the Jews in Spain, New York, 1968, p. 47, le responsum nº 16, $4^{\mathrm{e}}$ part. de Salomon Ben Adret montre qu'un chrétien pouvait porter plainte contre un juif devant la juridiction juive, et ainsi faire juger l'affaire par les juges
} 
III en 1284 à son justicier : « ...on soutient qu'entre un juif et un autre juif, on ne peut ni ne doit faire appel du droit hébraïque, nous vous demandons d'enquêter pour savoir si l'on peut faire appel... ». La lettre porte sur une affaire dans laquelle il y a eu mort d'homme, et dont les coupables veulent faire appel du jugement au roi. Leur requête suscite un débat de procédure en ce qui concerne la légitimité de l'appel $^{27}$. En effet, les parents de la victime ont fait savoir au roi que pour les affaires concernant des juifs, la coutume veut que ceux-ci ne puissent faire appel à d'autres qu'à des juifs.

La question de l'appel à une juridiction non juive est débattue entre juifs dans de nombreux endroits de la Chrétienté. À partir du moment où les juifs se voient conférer le pouvoir de rendre la justice, les autorités décisionnaires prétendent le conserver exclusivement à l'intérieur de la communauté ${ }^{28}$; il en va de la pérennité d'un privilège qui entérine une forme d'autonomie. Formellement pourtant, dans les territoires aragonais rien ne s'oppose à l'appel à une telle juridiction. En effet, officiellement rien ne l'interdit, on en veut pour preuve le très grand nombre de cas d'appels judiciaires formulés auprès du roi. Par ailleurs, contrairement à ce qui s'est produit dans les communautés juives d'Aschkenaze, les autorités rabbiniques d'Aragon n'ont pas entériné l'interdiction de l'appel formulée par "Rabbeinou Tam", grand halakhiste ashkénaze au XII ${ }^{\mathrm{e}}$ siècle, et qui est observée à peu près partout $^{29}$. Il y a donc, de fait, une ambiguïté juridique sur la question, ce qui explique la demande d'éclaircissement formulée par le roi auprès de son justicier. Le témoignage de Salomon Ben Adret à ce sujet est éloquent, il est extrêmement sévère à l'égard de ceux qui font appel des décisions prises par l'aljama devant les juridictions non juives. À un homme qui se plaint du jugement rendu par le Beth Din de Barcelone qu'il considère comme injuste, Rashba $^{30}$ répond que « la loi du Beth Din est vérité et (qu') aucune autre loi ne convient dès lors que les deux parties en conflit sont des juifs. Il n'y a aucune raison de recourir à la loi des non-juifs $»^{31}$. Les juifs qui ont recours à ce procédé sont souvent considérés comme des malshinim ou délateurs, la plaie de la société juive médiévale, comme les qualifient les

\footnotetext{
juifs en vertu de la loi juive. Pour certains chrétiens la justice juive est de meilleure qualité que la justice de la Couronne, ce qui explique qu'ils préfèrent s'en remettre à elle.

${ }^{27}$ BARCELOnE, ACA, Chancellerie royale, reg. 43, fol. 54v, Saragosse 05.11.1284, RÉGNÉ History of the Jews in Aragon, p. 221, $\mathrm{n}^{\circ}$ 1224: [...] asseratur quod inter iudeum et iudeum non potest nec debet iuxta cunam ebraicam appellare, mandamus vobis quot cognoscatis si appellari potuit [...]

${ }^{28}$ D. BIALE, Power and powerlessness, p. 53, explique que le fondement de l'interdiction de recourir aux tribunaux civils se trouve dans le Talmud de Babylone, traité Gittin 88b, Londres, 1990 : "Rabbi Tarfon avait coutume de dire : en tout lieu où vous trouvez des tribunaux civils, même si leur loi est la même que la loi juive, vous ne devez pas y avoir recours".

${ }^{29}$ Faire appel d'un jugement prononcé par un juge juif en vertu de la Halakha devant une juridiction non juive même royale - est assimilé à un sacrilège et est donc fermement prohibé par les autorités religieuses.

${ }^{30} \mathrm{C}$ 'est l'acronyme de RAbbi SHlomo Ben Adret, par lequel on a souvent coutume de l'appeler.

${ }^{31}$ RASHBA, Responsum n ${ }^{\circ} 239,2^{\mathrm{e}}$ part., éd. CD-Rom Responsa Project de l'Université Bar Ilan.
} 
rabbins et, à ce titre, ils encourent les peines les plus lourdes. Il existe une seule exception à cette règle, formulée par Salomon Ben Adret : «Il est interdit de conduire un juif ou une juive de notre communauté devant le capitoul, où il peut recevoir une peine corporelle ou financière...sauf pour les affaires de créance $»^{32}$. Cette exception est répétée à plusieurs reprises. Pour les autres cas toutefois, nous pouvons imaginer combien doit être grande la tentation, pour un condamné, de recourir à l'appel malgré l'interdit religieux, si une réduction de peine est possible. Avec le temps, le système des lettres de rémission se diffuse, même pour des crimes graves, avec pour contrepartie un monnayage conséquent. Dans un autre responsum, Salomon Ben Adret développe et justifie cet impératif du jugement en fonction de la loi juive pour les affaires entre juifs. En effet, dans la mesure où les juifs ont l'obligation de respecter la Halakha, seul le Beth Din qui la connaît et juge en vertu de ses principes, est habilité à juger : « les juifs doivent être jugés en fonction de la Torah, or les juges non juifs ne jugent pas en fonction de la Torah $»^{33}$. Un autre responsum de Salomon Ben Adret adressé à l'aljama de Lérida, se fait l'écho de cette disposition avec ces premiers mots : «La communauté a le pouvoir de la part du royaume de nommer deux ou trois hommes juifs qui jugeront les affaires entre juifs... $»^{34}$. Autrement dit, la répartition des responsabilités est bien comprise par le roi et par le détenteur de l'autorité rabbinique.

\section{2) Les enjeux royaux de la justice à l'égard des juifs.}

Malgré la confirmation de la responsabilité judiciaire des agents de l'aljama, des nonjuifs interviennent dans la justice rendue à des juifs et par des juifs. Les agents du roi interviennent notamment au moment de l'application des sanctions. Ces viguiers, bayles, justicia ou merinos ${ }^{35}$ exécutent les décisions judiciaires en accord avec la décision du Beth Din, infligent les peines et perçoivent les amendes fixées par le juge ${ }^{36}$. Nous avons vu que l'aljama de Lérida obtient le privilège de nommer deux ou trois personnes pour statuer dans les affaires civiles et criminelles dans lesquelles des juifs sont impliqués. Le responsum se poursuit par les mots suivants : «et ils - les hommes chargés de rendre la justice - seront

\footnotetext{
${ }^{32}$ ID. Responsum $\mathrm{n}^{\circ} 384,3^{\mathrm{e}}$ part., Ibid. Il y a concordance parfaite avec les règles édictées par les Fueros de Aragon et notamment l'article 121 qui précise la modalité des contrats de prêt conclu entre juifs et non-juifs.

${ }^{33}$ ID. Responsum $\mathrm{n}^{\circ} 254,6^{\mathrm{e}}$ part., Ibid.

${ }^{34}$ ID. Responsum $n^{\circ} 385,3^{\mathrm{e}}$ part., Ibid.

${ }^{35}$ Voir Porras Arboledas, Ramírez Vaquero, Sabaté i Curull, Historia de España, t. 8, p. 396-39. Le nom varie en fonction des territoires et en fonction de l'extension de leurs responsabilités. Cependant, ils exercent tous, peu ou prou, les mêmes fonctions. Et aussi Ibid. p. 390 : Implica el establecimiento de cortes jurisdiccionales en las capitales locales y regionales, donde se administre justicia como indicativo de la potestad por parte de un oficial dotado de ella por especifico nombramiento del soberano a quien representa.

${ }^{36}$ Voir Ibid. p. 391, donne une idée de la valeur des amendes puisque celles-ci représentent en moyenne un tiers des sommes en jeu dans le cadre des contentieux financiers.
} 
obligés de faire connaître au représentant du roi toutes les affaires qui parviendront devant eux, afin qu'il puisse percevoir les amendes fixées $\gg{ }^{37}$. Il y a donc partage des responsabilités et des compétences. Les responsa de Salomon Ben Adret nous apprennent par ailleurs que la communauté de Saragosse s'est vue concéder le privilège de juger les affaires civiles entre juifs et non-juifs, lorsque ces derniers sont les plaignants. Cependant les non-juifs ne sont pas obligés de se soumettre aux décisions adoptées par les juges juifs, à moins qu'ils aient prêté serment de le faire devant le notaire de la ville en présence de deux témoins ${ }^{38}$. Ce privilège est étendu par Pierre III en 1280 à toutes les aljamas catalanes. La répartition des rôles entre responsabilités juives et non juives dans les affaires de justice révèle un autre enjeu fondamental de la justice pour le roi : son enrichissement. Il est le produit de deux types de procédures : la première, régulière, consiste en la perception des amendes imposées au titre de la sanction judiciaire. Le roi étant le plus haut justicier, c'est lui qui perçoit les amendes ${ }^{39}$. Par ailleurs, les juifs lui étant liés par une relation particulière, c'est à double titre qu'il exerce à leur égard cette fonction de justice.

Christian Guilleré estime que "les revenus régaliens de l'exercice de la justice donnent un pourcentage relativement élevé : 15, 64\%. Lorsqu'un crime ou un délit commis est jugé par la justice royale (souvent en appel), la condamnation est souvent assortie d'une composition (diffinicio) en argent. Cette composition peut aussi bien porter sur les individus que sur les communautés, sur des délits de type féodal que sur des délits personnels ou même sur la mauvaise gestion d'une cité" ${ }^{40}$. Les différentes instances judiciaires se livrent une âpre concurrence pour les affaires concernant les non juifs et les amendes qui sont à la clér ${ }^{41}$. En revanche les juifs, lorsqu'ils sont justiciables à l'extérieur de la communauté, ne dépendent que de la justice royale. La régularité de la procédure veut que le roi perçoive les amendes fixées après condamnation par les juges de l'aljama, amendes qui alimentent ainsi les caisses du trésor royal. Mais l'éventail des peines est large et certains crimes requièrent des sanctions lourdes, c'est-à-dire des peines de prisons, de mutilation ou de mort. Et comme nous l'avons vu plus haut, c'est paradoxalement pour ces derniers cas que les profits semblent les plus

\footnotetext{
${ }^{37}$ RASHBA, Responsum $\mathrm{n}^{\circ} 385,3^{\mathrm{e}}$ part.

${ }^{38}$ Voir EPSTEIN, The responsa, p. 47, lequel renvoie à RASHBA, responsum $\mathrm{n}^{\circ} 16,4^{\mathrm{e}}$ part.

${ }^{39}$ Voir Porras Arboledas, Ramírez VAquero, SABAté i Curull, Historia de España, t. 8, p. 389-390 : La jurisdicción se interpreta como propia del soberano, el cual puede haber delegado su disfrute parcial o compeltamente -mero y mixto imperio, alta y baja jurisdicción- a los distintos poderes baroniales. De este modo, el detentor nato de la jurisdicción es el rey [...]

${ }^{40}$ Voir C. GuILlERÉ, Les finances dans la Couronne d'Aragon au début du XIV siècle (1300-1310), Estudios sobre renta, fiscalidad y finanzas en la Cataluña bajomedieval, ed. M. SÁNCHEZ MÁrTINEZ, Barcelone, 1993, p. 496.

${ }^{41}$ Voir n. 37.
} 
importants pour le trésor royal, par l'intermédiaire du droit de grâce ${ }^{42}$. En effet, le deuxième type de procédure qui conduit à l'enrichissement du roi pour des motifs judiciaires est extra ordinaire : il s'agit des procédures de rémission, qui se multiplient au cours de la période, et qui peuvent se révéler vraiment lucratives. On en retiendra deux exemples emblématiques, l'un individuel, l'autre collectif.

En 1285, deux juifs de Figueras Abraham de Turre et son fils Vidal, sont inculpés d'une multitude de crimes et délits, certains mineurs - comme d'avoir taillé les arbres d'un concitoyen -, d'autres beaucoup plus graves, allant jusqu'au meurtre. Abraham est notamment accusé d'avoir étouffé les deux enfants qu'il a eus d'une sarrasine; d'avoir entretenu à son domicile des relations coupables avec une autre; exigé de ses débiteurs des intérêts qu'ils lui avaient déjà versés; d'avoir conclu des contrats de prêt à des taux contraires à ceux fixés par la législation royale; de n'avoir pas payé ses impôts; d'avoir falsifié des documents; provoqué des rixes avec des juifs comme avec des chrétiens; d'avoir pénétré armé dans la synagogue de Figueras pendant l'office; d'avoir voulu tuer avec une épée un homme dans la synagogue le jour de Shabbat et d'en avoir extirpé un autre par les cheveux en l'insultant atrocement et même d'avoir voulu tuer son propre père et voler son frère ${ }^{+3}$. La liste continue et elle est longue. Même si nous savons que les faux témoignages sont courants pour mettre en cause des adversaires ou tirer profit d'une condamnation, la liste des motifs d'incrimination est ici tellement fournie, que nous avons du mal à croire à un faux témoignage qui justifierait par exemple la rémission royale ${ }^{4}$. Par ailleurs il n'en est absolument pas question dans la lettre adressée par l'infant Alphonse aux inculpés - suite à l'appel qu'ils ont formulé - pour motiver sa décision : «Abraham et Vital de Turre...nous vous absolvons, interrompons, et remettons définitivement de toute action, requête et demande et de toute peine civile et criminelle que nous pourrions demander contre vous, les vôtres ou vos biens $»^{45}$. Abraham de Turre et son fils

\footnotetext{
${ }^{42}$ C. Gauvard, De Grace especial. Crime, État et société en France à la fin du Moyen Âge, t. 1, Paris, 1991, p. 11 précise que "L'histoire des rapports entre l'État et le crime est celle d'une transmutation au terme de laquelle le pouvoir justicier peut s'octroyer le droit de punir ou de pardonner tout en restant objet d'amour. À cette opération d'ordre politique, il existe un témoin qui est aussi un garant, l'opinion publique que révèlent à la fois les obsessions des chroniques et les normes non écrites que le crime a bafouées ".

${ }^{43}$ BARCELONE, ACA, Chancellerie royale, reg. 62, fol. 136r-137r, Gérone 15.03.1285. RÉGNÉ, History of the Jews in Aragon, p. 238, n 1316.

${ }^{44}$ Voir GAUVARD, De Grace Especial, t. 1, p. 63 qui définit ce qu'est la rémission et les lettres qui la formalisent dans l'espace français aux $\mathrm{XIV}^{\mathrm{e}}$ et $\mathrm{XV}^{\mathrm{e}}$ siècles: "La lettre de rémission est un acte de Chancellerie par lequel le roi octroie son pardon à la suite d'un crime ou d'un délit, arrêtant ainsi le cours ordinaire de la justice, qu'elle soit royale, seigneuriale, urbaine ou ecclesiastique. Outre la remise de peine, l'accusé est pleinement rétabli dans sa bonne renommée et dans ses biens, les intérêts de la partie adverse étant néanmoins préservés".

${ }^{45}$ BARCElone, ACA, Chancellerie royale, reg. 62, ff. 136r-137r, Géroné 15.03.1285. RÉGNÉ, History of the Jews in Aragon, $\mathrm{n}^{\circ} 1316$ : [...] absoluimus definimus et remittimus vobis predictus Abrahe de Turri et Vitali [...] perpetuo omnem actionem, peticionem, et demandam et omnem penam civilem et criminalem quam contra vos
} 
sont donc totalement lavés des peines qu'ils encourraient du fait de leur inculpation. Plus loin dans la lettre est mentionnée la contrepartie de la rémission : «Nous reconnaissons avoir reçu de vous pour ces absolution, non-lieu et remise, la somme de sept mille quatre vingt dix sous barcelonais, sur laquelle nous nous sommes accordés selon votre bonne volonté ». Plus de 7000 sous sont ainsi venus enrichir le trésor royal pour la grâce de deux hommes qui avaient peu de chances de ne pas être condamnés. La dernière phrase nous renseigne précisément sur le processus de la rémission: il semble que dans le cadre de l'appel formulé au roi, le condamné a ouvertement proposé un marché - l'expression pactati sumus renvoie bien à cette notion de marché -, précisant même peut-être la somme qu'il était prêt à verser.

Le deuxième cas consiste en une rémission collective. Il s'agit de la grâce accordée à la suite de la condamnation pour hérésie d'un groupe de juifs de Calatayud. L'affaire se déroule sur plus d'un an de décembre 1341 à avril 1343 - et même sur plusieurs années si l'on observe les origines du dossier ${ }^{46}$. Elle met aux prises plusieurs intervenants : le groupe de juifs accusés d'hérésie, l'Inquisiteur général Bernard de Puycerda, le bayle de Calatayud, les délateurs des juifs, l'aljama de Calatayud, et enfin le roi. Sept documents permettent de suivre le déroulement de l'affaire en fournissant au fur et à mesure des informations de plus en plus détaillées sur les personnes concernées.

L'affaire nous est présentée dans une lettre de décembre 1341 : 19 personnes ont été accusées du crime d'hérésie ${ }^{47}$. Logiquement l'enquête après accusation a été confiée à l'inquisiteur de la province, le dominicain Bernard de Puycerda. Nous ne savons rien des accusateurs. Les documents emploient le terme de calumpniose pour qualifier l'accusation dont ce groupe de juifs fait l'objet. Ce mot renvoie-t-il à une accusation mensongère ou tout simplement à une dénonciation? Les deux possibilités sont envisageables, de plus il y a souvent superposition entre calomnie et délation. Quoi qu'il en soit, le groupe de juifs a bel et bien été condamné. Or le roi lui fait remise de la condamnation. En effet, le paiement des

\footnotetext{
vel altrum vestrum aut bona vestra possemus facere [...] puis : Pro hac absolutione, definicione, remissione concedimus a vobis habuisse recepisse septem mille nonagentos soli barchinone de quibus bonum pactati sumus ad vestrum voluntatem $[\ldots]$

${ }^{46}$ Un document de 1328 parle déjà de l'affaire. Abraham de Quatorze a été incarcéré avec sa femme à la suite de l'enquête menée contre lui pour hérésie. BARCELONE, ACA, Chancellerie royale, reg. 432, fol. 175r, Montblanch, 21.07.1328.

${ }^{47}$ Ibid. reg. 621, fol. 190r, Valence, 18.12.1341. : Salomon Passariel, Brahem Mocatil, Samuel Alpastan, Salomon Avensaprut, Josef Paragon, Mosse Abnalguer, David Avenrodach, Acach Avenhalez, Rabin Salomon fils de Rabin Mair, Cadoch Alieceni, Sabach del Saig, Farcon Abenafia, Mayr Abnalguer, Josef Almadeya, Abrahim Azarias, Josef Alconstanti, Mosse Abenforna, Josef Bavini, Mosse Paragon. Le document reprend les motifs : composicionem fecerint cum curia nostra pro quantitate viginti unius mille soli regalium ratione remissionis per nos eis facte de eorum bonis quae dicebant nostre curie confiscata occasione inquisitionis quam frater Bernard [...] faciebat [...]
} 
21000 sous n'est pas la peine pécunière sanctionnant la condamnation pour hérésie, mais la contrepartie de sa rémission. En première instance, ce groupe de juifs a été condamné à la « confiscation » pure et simple « de ses biens ». C'est donc une amélioration de leur sort, que ce paiement de 21000 sous. Le second document du dossier nous apprend par ailleurs que le groupe avait « fui au royaume de Castille » après la condamnation, pour échapper à ses effets. Il y a donc eu, en quelque sorte, monnayage de son retour ${ }^{\text {s. }}$.

On voit avec cette affaire -dont nous avons brièvement résumé le canevas et le processus de résolution- l'imbrication des acteurs et la complexité des relations qu'ils entretiennent, qui interviennent dans le déroulement de la procédure judiciaire. Son cours peut être sinueux, entravé par des manœuvres dilatoires ou carrément délictueuses, qui mènent à des situations aberrantes pour les justiciables. Ces éléments montrent également la fragilité du consensus dégagé par les juifs et le roi autour de la justice, qui peut être mis à mal de diverses manières.

\section{Les entraves au compromis}

Le compromis trouvé par les juifs et le pouvoir royal autour de l' « administration de la justice » se trouve parfois mis en péril par l'intervention de deux agents : la prétention à juger des juridictions concurrentes du roi et l'irruption dans le « jeu » judiciaire de ceux que l'on pourrait qualifier de « trouble-fête » du consensus: les malshinim.

1) Les tentatives de contournement de la justice royale

On n'insistera pas ici sur les prétentions féodales à la justice rendue pour les juifs. On a dit plus haut que le domaine réservé qu'elle constitue pour le roi, comme tout ce qui ressortit aux affaires juives, est accepté, bon gré mal gré, par les autres acteurs de la société aragonaise. En revanche, l'Église fait valoir sa légitimité à juger des affaires impliquant des juifs par un nouveau biais à partir de la fin du XIII ${ }^{\mathrm{e}}$ siècle : l'Inquisition.

La seule façon pour l'Église d'en finir avec ses ennemis véritables ou supposés -les juifs refusant de reconnaître en Jésus le Messie en font partie- est en effet de les accuser $\mathrm{d}^{\prime}$ hérésie ${ }^{49}$. L'hérésie est le plus grand crime possible contre l'orthodoxie chrétienne; elle suppose l'éradication de ceux qui en sont coupables ${ }^{50}$. Celle-ci se traduit soit par l'élimination

\footnotetext{
${ }^{48}$ Ibid. fol. 188v, Valence 17.12.1341 : [...] contra eos super crimine heretice pravitatis de quo calumpniose ut fertur extiterant accusati in accipiendo fugam ad regnum Castille [...]

${ }^{49}$ Voir J. Trachtenberg, The Devil and the Jews. The Medieval Conception of the Jew and its Relation to Modern Antisemistism, Philadelphia-Jerusalem, 1983, rééd. 1993. Le chap. 12 analyse le passage de l'accusation d'infidélité formulée à l'encontre des juifs à celle d'hérésie.

${ }^{50}$ Avant d'arriver à cette extrémité, l'excommunication a un vrai pouvoir coercitif, voir E. VoDOLA, Excommunication in the Middle Ages, Berkeley-Los Angeles-London, 1986, p. 191.
} 
pure et simple si les inculpés refusent d'abjurer leur hérésie, soit par la conversion à l'orthodoxie s'ils l'acceptent. À partir de 1250 l'Inquisition confiée aux Dominicains, chargés de débusquer et redresser l'hérésie, est bien définie. Au départ les infidèles juifs et musulmans n'en sont pas la cible. Vers ceux-ci, il convient de développer un apostolat particulier, mais qui n'est pas le fait de l'Inquisition. Or au XIII ${ }^{\mathrm{e}}$ et surtout durant la première moitié du XIV siècle se produit un changement qualitatif d'approche du judaïsme par l'Inquisition. D'après Anna Foa, les inquisiteurs s'efforcent d'étendre leur juridiction aux juifs en levant contre eux des charges d'hérésie ${ }^{51}$ ayant pour objectif leur conversion. La religion juive jusque-là tolérée devient suspecte. Sont visées non pas ses fondements doctrinaux, mais certaines de ses pratiques. Il ne s'agit donc pas d'une attaque globale portant sur le fond, mais d'attaques ponctuelles concernant la forme.

Deux points sont susceptibles de cristalliser l'instruction par l'Inquisition de dossiers d'hérésie juive : le premier point regroupe des attitudes contraires au christianisme : pratiques magiques, actes contraires à l'orthodoxie chrétienne ou encore blasphème ${ }^{52}$. Le deuxième point concerne la conversion de chrétiens au judaïsme ou l'apostasie de convertis qui ont fait retour au judaïsme. Ainsi, au XIII ${ }^{\mathrm{e}}$ et aux $\mathrm{XIV}^{\mathrm{e}}$ siècles, l'Église assimile la sorcellerie, définie comme le recours aux puissances occultes et la fréquentation du diable, à l'hérésie. Cette définition atteint les juifs également comme on le voit à travers la promulgation de l'Apparatus d'Innocent IV en 1254, qui permet au Pape de les juger lorsqu'ils commettent des hérésies contre leur propre religion ${ }^{53}$. L'instrument de cette incrimination est le Talmud, considéré comme un ouvrage de magie, et ses lecteurs comme des invocateurs de démons. Cela explique la multiplication dans la seconde moitié du XIII ${ }^{\mathrm{e}}$ siècle, des accusations de blasphème portées contre le Talmud, qui débouchent par exemple dans le royaume de France sur la crémation de centaines d'exemplaires de cette oeuvre ${ }^{54}$. Le principal objet de cette

\footnotetext{
${ }^{51}$ A. FoA, The Witch and the Jew : Two Alikes that were not the same, From Witness to Witchcraft, Jews and Judaism in Medieval Christian Thought, éd J. COHEN, Wiesbaden, 1996, p. 361-374.

${ }^{52}$ Voir C. LEVELEUX, La parole interdite, le blasphème dans la France médiévale XIII -XIVe siècles : Du péché au crime, Paris, 2001, p. 226. Les rites juifs sont mis en accusation en même temps que le fonds des écrits pour condamner certains éléments du judaïsme. Il est question d'attitudes (le port de costumes et la célébration de danses) et de propos (des passages du Talmud) jugés offensants pour le christianisme. On retrouve là peut-être la confusion observée plus haut entre des rites spécifiques tel celui de Purim et des temps sacrés de la liturgie juive d'une part et chrétienne d'autre part.

${ }^{53}$ Voir J. Muldoon, Popes Lawyers and Infidels, The Church and the non christian World, Philadelphie, 1979, p. 10 : "Le Pape pouvait juger les juifs quand ils violaient la loi de l'Ancien Testament si leurs propres chefs n'appliquaient pas la loi. Le Pape était même responsable de l'orthodoxie de la doctrine religieuse juive. Innocent fit remarquer que lui et son prédécesseur Grégoire IX avaient ordonné la crémation publique du Talmud parce que "ces volumes contenaient de nombreuses hérésies".

${ }^{54}$ Voir A. TUILIER, La condamnation du Talmud par les maîtres universitaires parisiens, ses causes et ses conséquences politiques et idéologiques, Le brûlement du Talmud à Paris 1242-1244, dir. G. DAHAN, Paris, 1999, p. 76. Dans l'espace aragonais, les choses sont un peu différentes : le Talmud fait l'objet de suspicions et
} 
formulation est de permettre aux autorités de l'Église d'exercer un contrôle sur la littérature juive $^{55}$. Par la suite, la bulle Super illius specula, en 1326, charge l'Inquisition de l'instruction des affaires de magie. Les juifs en sont de plus en plus souvent accusés, ce qui permet de faire le lien plus facilement entre l'Inquisition et le traitement des affaires juives. Petit à petit se développe toute une littérature sur la question, assimilant les juifs et les musulmans aux invocateurs de démons, et donc à l'hérésie. L'aboutissement de cette logique est l'ouvrage consacré à ce thème par Nicolas Eymeric, dans les années $1370^{56}$, dont une conclusion est explicite : «La 3ème conclusion ou cause est que ce juif qui invoque les démons dans sa loi, est hérétique ».

Quant au deuxième point susceptible de provoquer une enquête inquisitoriale, si le judaïsme en lui-même n'est pas une hérésie, la conversion au judaïsme étant un dévoiement par rapport à la vraie foi, en constitue bien une. Nicolas Eymeric expose ainsi : « il est un cas selon lequel un juif séduit un chrétien, qui à la fin se convertit aux juifs, ce en quoi il peut être poursuivi par l'Inquisition contre le juif $\rangle^{57}$. Les conversions au judaïsme provoquent les réactions indignées du clergé ; le Pape fulmine en 1267 la bulle Turbato corde adressée aux Ordres Mendiants : « ... ces juifs qui ont conduit jusqu'ici des chrétiens des deux sexes à leurs rites, que vous les ameniez à l'encontre de cela, ou que vous les en punissiez par des amendes $»^{58}$.

Observons des exemples de cas traités par l'Inquisition. Tous les dossiers impliquant des juifs, à une exception près, datent de la période postérieure aux années 1320 avec un pic dans les années 1323-1326, 1337 et 1342-1348.

d'enquêtes après la Dispute de Barcelone en 1263, mais les conséquences ne sont pas aussi dramatiques qu'elles l'ont été dans le royaume de France.

${ }^{55}$ Voir G. DAhan, Les juifs dans le Commentaire du Décret de Gui Terré, Homenaje al Prof. David Romano Ventura, Sefarad 52/2, 1992, p. 396, précise que "C'est au XIII siècle qu'apparaît le thème de l'hérésie des juifs ; mais, en réalité il n'y a jamais confusion entre juifs et hérétiques chrétiens ; si les juifs apparaissent comme hérétiques, c'est par rapport à leur propre loi [...] Gui Terré [...] fait subir au thème une mutation profonde; la liste des erreurs des juifs par rapport aux préceptes de l'Ancien Testament rejoint en fait l'interprétation chrétienne de celui-ci [...] De la sorte le judaïsme peut bien apparaître comme une hérésie chrétienne [...]".

${ }^{56}$ Nicolas EYMERIC, Tractatus contra daemonum invocatores, Bibliothèque nationale de France, Ms. lat. 14533, fol. $95 \mathrm{vb}$.

${ }_{57}^{57}$ Ibid., fol. 96 ra.

${ }^{58}$ Bulle du 27.07.1267 adressée aux inquisiteurs dominicains et franciscains, éditée dans S. SimONSOHN, The Apostolic See and the Jews, Documents, 492-1404, Toronto, 1988, p. 236-237.

${ }^{59}$ Y.T. Assis, Juifs de France réfugiés en Aragon (XIII ${ }^{\mathrm{e}}$-XIV ${ }^{\mathrm{e}}$ siècles), Revue des Études Juives, t. 142, 1983, p. 285-322, met en relation la forte intensification des menées inquisitoriales contre les juifs en Aragon avec l'accueil dans ces territoires de juifs expulsés de France et qui ont dû se convertir au christianisme avant de s'enfuir. Ainsi l'Inquisition ne s'en prend-elle pas à n'importe quels juifs mais à des convertis qui, constatant le sort bien plus favorable fait aux juifs en Aragon, décident une fois réfugiés de revenir au judaïsme, ce qui justifie l'action inquisitoriale. Voir aussi, A.T. CALDERS, Los judios y la Inquisicion catalana (siglos XII-XIV), El Olivo 5/6, 1978, p. 51-60. 
En mars 1326, des juifs de l'aljama de Calatayud, sont incriminés pour possession d' ouvrages suspects qui ont été confisqués par l'inquisiteur de la province, Raymond de Miedes, franciscain. Celui-ci a en effet réclamé qu'on lui remette les livres confisqués aux juifs considérés comme hérétiques, ces livres pouvant être utiles à l'ordre. Il s'agit d'une Bible en hébreu, un livre vocatum Sarasin, et des livres du Talmud ${ }^{6}$. On peut souligner le fait que les juifs sont accusés d'hérésie pour avoir possédé ces livres, mais que les Mineurs estiment pouvoir en faire bon usage.

En décembre 1326 se présente un cas similaire : la confiscation à des juifs de Calatayud une fois de plus, de leurs livres, duo midrasses - traités du Talmud -, par l'inquisiteur Guillaume Costa ${ }^{\iota}$. Les similitudes entre les deux cas révèlent peut-être en fait une seule et même affaire. Quoi qu'il en soit, nous sommes bien en présence d'une enquête menée par l'Inquisition, sinon directement au motif d'hérésie, du moins pour la possession d'ouvrages considérés comme hérétiques. On reconnaît dans la suspicion avec laquelle on considère le Talmud, l'opinion qui avait prévalu et s'était diffusée en France à la suite de la Dispute de Paris en 1242, même si dans l'espace aragonais les positions sur la question sont moins tranchées. Par ailleurs, on voit bien ici comment le Talmud constitue l'instrument « idéal » pour l'incrimination des juifs et de ce fait permet la transition vers la rupture du « consensus augustinien ».

Un autre exemple soulève de nombreuses questions: il s'agit de l'enquête mentionnée plus haut- diligentée contre un groupe de juifs de Calatayud auxquels le roi remet leur peine contre le versement d'une somme d'argent importante ${ }^{\omega_{2}}$. Il est intéressant ici d'analyser le dossier non pas dans sa dimension pécunière, mais au prisme de l'idéologie et du facteur religieux. Ceux-ci permettent de mettre en évidence le rapport de force entre le roi et l'Église à travers les tentatives de l'Inquisition pour incriminer les juifs et la protection royale monnayée pour les disculper. En fin de compte, la justice royale l'emporte puisque les juifs sont grâciés. Mais cette justice peut toutefois être mise à mal d'une autre façon.

\section{2) Le malshin ou la rupture du compromis.}

\footnotetext{
${ }^{60}$ BARCELONE, ACA, Chancellerie royale, reg. 285, fol. 172r, Barcelone 20.03.1326.

${ }^{61}$ Ibid., reg. 249 fol. 277, Barcelone 25.12.1326 ; RÉGNÉ, History of the Jews in Aragon, p. 624, n 3413.

${ }^{62}$ BARCELOnE, ACA, Chancellerie royale, reg. 223, fol. 227r, Barcelone 18.03.1323, RÉGNÉ n 3256 ; ACA reg. 248 fol. 28r, Barcelone 22.09.1323, RÉGNÉ n 3276 ; ACA reg. 621 fol. 188v, Valence 18.12.1341; fol. 190r-v, même lieu, même date.
} 
Bien qu'évoquée plus haut, il faut revenir sur la question des malshinim, ou malshinout, en ce qu'elle apparaît cruciale dans les relations entre le pouvoir royal et la minorité juive. Il semble que se joue à travers cette question bien plus que le faux témoignage ou la dénonciation, et la fréquence de sa mention dans les ordonnances rabbiniques comme dans les décisions royales reflète sa sensibilité ${ }^{63}$. Dans un responsum adressé par Salomon Ben Adret à la communauté de Lérida, nous apprenons qu'un homme s'est plaint aux Berourim - les agents - de cette aljama d' «avoir été dénoncé en dépit de la Tachana sur la malshinout décrétée par la communauté alors qu'il est écrit dans la Tachana : il n'est pas permis de conduire un juif ou une juive de notre communauté devant le capitoul où il peut recevoir une peine corporelle ou une peine financière...sauf pour les affaires de créance $»^{64}$. La question préoccupe Salomon Ben Adret qui y revient à plusieurs reprises. Dans un autre responsum, il expose la nécessité de définir plus précisément le fond de la Taqana : « ...il y a des plaintes devant les tribunaux étrangers - la justice civile - et la protection contre la malshinout est annihilée et l'argent des juifs est dépensé dans des affaires sans nécessité. De ce fait la communauté a décidé de prendre des mesures afin d'empêcher ces dérives » ${ }^{65}$. L'appellation générique de malshinout recouvre plusieurs types de délit possibles, allant du plus bénin : la dénonciation d'un manquement véritable, au plus pernicieux : la fausse accusation. D'une manière générale, considéré par les autorités rabbiniques comme plus grave encore que le meurtre, le crime de malshinout est puni plus lourdement ${ }^{66}$. L'accusation mensongère constitue le cas le plus lourd de malshinout. Pour comprendre la portée de ce délit, il faut se rappeler la valeur de la parole et de la réputation dans la société médiévale.

\footnotetext{
${ }^{63}$ Voir à cet égard les articles d'E. LourIE, Mafiosi and Malsines : Violence, Fear and Faction in the Jewish Aljamas of Valencia in the Fourteenth Century, Crusade and Colonisation: Muslims, Christians and Jews in Medieval Aragon, éd. E. LOURIE, Aldershot, 1990, Essay XII, p. 69-102 ; M.D. MEYERSON, Revisiting the WaxPress Affair in Morvedre (1326-27): Jewish Fiscal Politics in the Kingdom of Valencia, Jews, Muslims and Christians in and around the Crown of Aragon, Essays in Honour of Prof. Elena Lourie, éd. H.J. HAMES, LeydeBoston, 2004, p. 303-320. Ces deux articles élargissent de façon passionnante les perspectives d'analyse de la malshinout montrant en quoi elle peut être une arme politique, non seulement entre les mains des véritables malshinim, mais aussi lorsqu'une telle accusation est instrumentalisée contre un adversaire. Etre vraiment malshin ou bien faire l'objet d'une dénonciation volontairement calomnieuse de malshinout entraine des conséquences désastreuses.

${ }^{64}$ RASHBA, Responsum n ${ }^{\circ} 384,3^{\mathrm{e}}$ part. éd. CD-Rom Responsa Project de l'Université Bar Ilan.

${ }^{65}$ Ibid. $\mathrm{n}^{\circ} 297,5^{\mathrm{e}}$ part.

${ }^{66}$ L'Encyclopaedia Judaica fait remonter cette attitude ou opinion à l'époque talmudique. La position du Talmud à l'égard des malshinim ou informateurs est très hostile. Il est dit d'eux : "Ils descendront jusqu'à la Géhenne pour y être punis jusqu'à la fin des générations" (Traité Rosh Ha-Shana 17 a, Londres 1990). Le problème est encore plus grave au Moyen Âge du fait des dangers qui pèsent sur la société juive. La révélation aux non-juifs de choses secrètes quant à l'organisation sociale et politique de ces communautés risquait de mettre en danger leur autonomie. C'est ce danger socio-politique qui incita les Sages à adopter une sévérité qui n'était pas coutumière à l'esprit talmudique. De la sorte alors que depuis la disparition du Sanhedrin, la justice juive ne pouvait en théorie prononcer de sentence de mort, les cas de malshinout constituent une exception et de telles sentences ont été prononcées en divers lieux de la chrétienté.
} 
Pour les juifs comme pour les non juifs, la fama constitue une référence primordiale ; être diffamé débouche souvent sur la perte de l'honneur ${ }^{67}$. Celle-ci a des conséquences sociales plus lourdes encore: la perte de la confiance et de ce fait le début de l'isolement. C'est donc l'ordre social dans son entier qui est en jeu en cas de diffamation. Or ces cas se multiplient au cours de la période analysée pour satisfaire des intérêts divers : par exemple pour mettre en doute l'honnêteté de tel personnage important et donc fragiliser sa position sociale. La jalousie, l'ambition, mais aussi le durcissement des relations à l'intérieur d'une société - juive comme chrétienne - de plus en plus hiérarchisée, peuvent être les moteurs de ces agissements. De ce fait, les malshinim sont considérés comme la plaie de la société juive médiévale. Pour tenter de limiter le phénomène, les autorités rabbiniques adoptent les mesures les plus strictes. Les malshinim, doivent être traités en parias et en hors-la-loi, on ne doit leur manifester aucune pitié. Salomon Ben Adret nous apprend que les responsables des communautés de Catalogne se sont réunis pour statuer sur la question car « les malshinim sont nombreux ${ }^{68}$. Rashba est catégorique : « il est licite de tuer un malshin ». Il ajoute que la peine de mort doit leur être appliquée sans hésitation ${ }^{69}$. Ben Adret précise même que les aljamas d'Aragon et de Catalogne devraient assurer l'exécution de ces peines « de leurs propres mains comme cela se fait tous les jours en de nombreux lieux d'Israël... ou en Castille, conduits à le faire par les sages de chaque génération ». Or dans la Couronne d'Aragon, elles ne le peuvent sans l'accord du pouvoir temporel. Les communautés réunies pour décider des mesures à prendre ont demandé au roi à bénéficier de ce droit.

S'ils sont considérés comme un fléau par les communautés juives, le pouvoir royal est lui aussi sensible à la capacité de nuisance des malshinim. Une lettre adressée par Alphonse IV à l'un de ses juges en 1328 l'informe que les membres de l'aljama d'Alagon : « ...ont fait

\footnotetext{
${ }^{67}$ À propos de l'honneur et de la Fama, voir J. PitT-RIVERS, Anthropologie de l'honneur. La mésaventure de Sichem, Paris, 1983, p. 18 : 'L'honneur est la valeur qu'une personne possède à ses propres yeux mais c'est aussi ce qu'elle vaut au regard de ceux qui constituent sa société. C'est le prix auquel elle s'estime, l'orgueil auquel elle prétend, en même temps que la confirmation de cette revendication par la reconnaissance sociale de son excellence et de son droit à la fierté". Voir aussi C. GAUVARD, La Fama une parole fondatrice. Médiévales, t. 24, 1993, p. 5-13; N. GONTHIER, Mala Fama et Honneste Conversation, Les critères de la morale populaire d'après les sources judiciaires aux XIV ${ }^{\mathrm{e}}$ et $\mathrm{XV}^{\mathrm{e}}$ siècles, Ordre moral et délinquance de l'antiquité au XX $X^{e}$ siècle. éd. B. GARNOT, Dijon, 1992, p. 33-46.

${ }^{68}$ Décision de Rashba sur la machinout en partie éd. CD-Rom Responsa Project de l'Université Bar Ilan. Voir A. Blasco Martínez, Los malsines del Reino de Aragón, una aproximación, Proceedings of the Eleventh World Congress of Jewish Stuies, June 22-29, 1993, Jérusalem, 1994, p. 83.

${ }^{69}$ D. Kaufman, Jewish informers in the Middle Ages, The Jewish Quaterly Review, t. 8, 1895-1896, explique qu'il existe une véritable juridiction d'exception en ce qui concerne les malshinim, au regard de la législation et des procédures habituelles. Ainsi dans le cas des malshinim il était possible de punir les coupables sans les avoir informés au préalable des peines encourues. De même il était possible de les juger par contumace ce qui était impossible pour les autres cas de procès. L'auteur justifie cette sévérité en expliquant qu'elle constituait une mesure sociale nécessaire dans une société médiévale volontiers hostile aux juifs. Il explique que le malshin venait encore renforcer le danger qui pesait naturellement sur eux et que, de ce fait, il était inacceptable.
} 
et décidé une certaine tachana ou document publique... selon laquelle si un juif a proféré une fausse accusation criminelle ou civile, les adelantati de l'aljama sont requis sous trois jours pour faire les enquêtes que nécessite une telle accusation $\rangle^{70}$. Le caractère officiel de la procédure suivie en cas de fausse accusation, la règle entérinée par la taqana ${ }^{71}$ et l'enquête menée par les adelantati, montrent que l'affaire est prise au sérieux. Le roi légifère et relaie les mesures adoptées par la communauté auprès de ses propres agents chargés de la justice, afin d'essayer de limiter leur action. Nous pouvons souligner que les cas de malshinout évoqués par la documentation non juive, datent pour la plupart de la fin de la période analysée. Ceci peut être mis en relation avec les tensions sociales croissantes à l'intérieur de la communauté. Les mesures prises par l'aljama d'Alagon faisaient suite à la mise en accusation mensongère d'un chirurgien, mais la calomnie peut viser n'importe qui. Une autre lettre d'Alphonse IV nous en donne un exemple en 1329, à propos d'un certain Salomon, juif de Barbastro, faussement accusé de meurtre et condamné pour cela à la confiscation de tous ses biens, mais surtout à l'infamie publique ${ }^{72}$. En 1333 à nouveau, le roi rend compte de la marche à suivre dans les affaires d'accusation pour éviter la diffamation. Il rappelle que, dès qu'une accusation est prononcée, l'aljama doit constituer une commission ad hoc, pour vérifier sa véracité. La procédure est extrêmement codifiée et doit permettre d'éviter les erreurs judiciaires, lourdes de conséquences ${ }^{73}$.

La convergence d'intérêts entre la minorité juive et le roi à propos de ces affaires apparaît à travers plusieurs biais. Les lettres de Pierre IV mentionnent explicitement le terme de malshin alors que jusque-là le délit et son auteur étaient désignés par des termes latins. Les peines qui le sanctionnent sont également mentionnées. Il n'est pas question de la peine de mort, mais pour un premier cas, en 1340, d'une amende de 1000 sous barcelonais à verser au trésor royal. Nous apprenons dans la même lettre que l'aljama de Barcelone a demandé au roi de faire en sorte qu'une fois l'amende payée, les personnes coupables de malshinout, « ne soient pas relâchées, mais gardées par le représentant du roi, afin que l'aljama mène sa propre

\footnotetext{
${ }^{70}$ BARCELOnE, ACAChancellerie Royale, reg. 431, fol. 241r, Daroca 26.08.1328 : [...] fecerunt et ordinarunt quandam tachanam sive publicum instrumentum in quo obligarunt se cum alatma et sub obligatione bonorum suorum quod si alicui judeo fieret aliqua falsa accusatio criminalis aut civilis quod infra tres dies ex quo essent requisiti adelantati dicte aliame haberent facere missiones necessarias tali accusato.

${ }^{71}$ On reprend dans les citations l'orthographe telle qu'elle figure dans les documents : ainsi tachana pour taqana ou malsin/malsinout/malsins pour malshin/malshinout/malshinim. En revanche, dans le corps de l'article, on emploie l'orthographe conventionnelle.

72 Ibid., reg. 436, ff. 209v-210, Valence 17.01.1329.

${ }^{73}$ Ibid., reg. 459, fol. 271v, Saragosse 17.10.1333 : [...] aliama iudeorum dicti loci fecerunt et ordinarunt quandam tacanam sive publicum instrumentum in quo obligarunt se cum alatma et sub obligatione bonorum suorum quod si alicui iudeo fieret aliqua falsa accusatio criminalis aut civilis quod infra tres dies ex quo essent requisiti adelantati dicte aliame haberent facere missiones necessarias.
} 
enquête ». Il y a donc collaboration entre l'autorité royale et les responsables communautaires pour gérer ce problème ${ }^{74}$. En 1342, la flagellation publique est prescrite comme sanction au crime de malshinout dont est donnée la définition suivante : la prévarication obtenue par des accusations calomnieuses. Dans la même lettre est également décrite la procédure qui s'ensuit du côté juif : la proclamation de la condamnation à la synagogue et l'expulsion du criminel, en l'occurrence Vitale Peperci, un juif de Saragosse ${ }^{75}$. Le compte-rendu d'un cas en 1345, précise que les calomniateurs espéraient tirer un profit financier des accusations qu'ils avaient lancées. Ils sont doublement coupables : d'abord parce qu'un juif a l'interdiction de traduire devant une autre justice que la justice juive un de ses coreligionnaires, ensuite parce qu'un malshin est par définition coupable d'une accusation mensongère ${ }^{76}$. Nous voyons donc à travers cette multiplication des documents et leur précision croissante, que cette question est fondamentale, non seulement pour la minorité juive, mais également pour l'autorité royale. Pour l'entité juive, il s'agit sans doute de ne pas se voir confisquer son privilège juridictionnel, ainsi que le symbole d'autorité et d'autonomie qu'il suppose. Quant au pouvoir royal, son intérêt pour cette question s'explique sans doute par le désir d'affirmer sa fonction de pouvoir justicier. La souveraineté royale s'exprime entre autres par l'exercice de la justice, et le roi ne doit pas être dupé. Or une accusation mensongère, une calomnie, si elles mettent la justice en branle, le font à mauvais escient et la mettent en défaut. La justice royale doit déboucher sur la proclamation de la vérité, elle ne peut être abusée. Le malshin, par ses mensonges, est fauteur de désordre, il met en péril l'équilibre précaire sur lequel se sont accordés le roi et la minorité juive, il brouille l'image que se renvoient l'un et l'autre, en y ajoutant les écrans de ses affabulations ${ }^{77}$. Le malshin contrevient deux fois à la loi : à la Halakha, loi de sa communauté et à celle du souverain dont il est un sujet.

\section{Conclusion : Des justices concurrentes ?}

\footnotetext{
74 Ibid., reg. 612 fol. 24r-v, Barcelone 10.12.1340. On peut rappeler la définition du terme malshin donnée dans ce document par la chancellerie royale: [...] ille iudeus qui accusator existeret qui in hebrayco malsin vocaretur.

75 Ibid., reg. 620 fol. 241r, Barcelone 27.02.1342.

76 Ibid., reg. 638 fol. 128 r, Barcelone 10.02.1345.

${ }^{77}$ Il semble que la situation ne s'arrange pas au cours de la période. D'après D. KAUFMAN, Jewish informers, p. 218, malgré les recommandations et les mises en garde de Rashba quelques décennies plus tôt, les représentants des communautés de Catalogne et du Royaume de Valence éprouvent la nécessité d'adopter un statut, le 25 septembre 1354, qui fait de l'extirpation des malshinim une affaire publique pour laquelle des taxes doivent être prélevées.
} 
Il ressort de cette analyse que le principal enjeu que recèle la question de la justice royale envers les juifs est celui de l'autorité : autorité supérieure du roi par rapport aux autres prétendants à la justice dans ses territoires, autorité des instances de l'aljama sur ses membres pour les affaires internes. La reconnaissance et l'acceptation de ces deux autorités par ceux qui en sont à la fois les témoins et les objets, sont les garantes de la perpétuation de la bonne entente entre le roi et ses sujets juifs. Cette entente est primordiale et tous en sont conscients comme l'attestent les précautions prises lorsque se présentent des situations délicates. En 1281, le roi Pierre III demande au bayle d'Huesca de faire connaître la procédure en ce qui concerne la condamnation d'un certain Isach Alcutaus, jugé par plusieurs autorités, les juges de l'aljama et la justice civile. Il lui demande de lui faire savoir laquelle des sanctions faire appliquer si l'une est plus lourde que l'autre et notamment si la peine appliquée en fonction de la loi des juifs est plus sévère ${ }^{78}$. On en déduit qu'en dépit des règles, Isach Alcutaus a dû faire appel à la justice civile. Par ailleurs, Salomon Ben Adret évoque le cas d'un homme convoqué devant le Beth Din pour être jugé et qui refuse de se présenter, voulant être jugé par la justice civile. Il rappelle l'interdiction qui pèse sur ce recours, mais ajoute que si toutefois le tribunal des non-juifs donne une sentence équivalant à celle qu'aurait donné le Beth Din, alors la sentence est considérée comme valide ${ }^{79}$. Le responsum de Rashba fait écho de façon frappante à l'interrogation du roi, les deux justices et les deux autorités sont soucieuses l'une de l'autre, tenant compte des limites fixées par les règles de coexistence, des latitudes autorisées, des prérogatives accordées, ainsi que de la sujétion due. L'équilibre entre les deux partenaires est donc bien le résultat d'une vigilance constante et de procédures précises. C'est en fonction de la Halakha que les juifs chargés de la justice exercent leur charge, malgré cela, le roi reste le juge suprême de toutes les affaires. Cet équilibre parfois fragile, cette complémentarité de fonctionnement donnent à l'existence juive dans l'espace aragonais son caractère exceptionnel.

\footnotetext{
78 Ibid., reg. 49 fol. 95r, Alcira 01.06.1281, RÉGNÉ, History of the Jews in Aragon, p. 156, n 866.

79 RASHBA, Responsum $\mathrm{n}^{\circ} 171,5^{\mathrm{e}}$ part., éd. CD-Rom Responsa Project de l'Université Bar Ilan.
} 